\title{
An analytical solution for response of a porous seabed to combined wave and current loading
}

\author{
Y. Zhang a,*, D.-S. Jeng ${ }^{\mathrm{a}, \mathrm{b}}$, F.P. Gao ${ }^{\mathrm{c}}$, J.-S. Zhang ${ }^{\mathrm{d}}$ \\ a Center for Marine Geotechnical Engineering, Department of Civil Engineering, Key Laboratory of Ocean Engineering, Shanghai Jiao Tong University, Shanghai 200240, China \\ ${ }^{\mathrm{b}}$ Division of Civil Engineering, University of Dundee, Dundee, DD1 4HN, UK \\ ' Institute of Mechanics, Chinese Academy of Sciences, Beijing 100190, China \\ d State Key Laboratory of Hydrology-Water Resources and Hydraulic Engineering, Hohai University, Nanjing 210098, China
}

\section{A R T I C L E I N F O}

\section{Article history:}

Received 29 March 2012

Accepted 17 September 2012

Available online 3 November 2012

Keywords:

Pore pressure

Wave-current interactions

Porous seabed

Effective stresses

\begin{abstract}
A B S T R A C T
In this paper, an analytical approximation for the evaluation of the pore pressure and effective stresses in marine sediments under combined wave and current loadings is derived. Unlike previous investigations, non-linear interactions between waves and currents are considered in this study. An analytical solution for the wave-current induced oscillatory soil response in marine sediment is presented first. Based on the proposed analytical solution, a parametric study for the liquefaction potential will be carried out. Parametric study results indicate that the influence of current and non-linear waves on the maximum liquefaction depth is significant.
\end{abstract}

(c) 2012 Elsevier Ltd. All rights reserved.

\section{Introduction}

When waves/currents propagate over the ocean, they exert fluctuations of dynamic pressures on the sea floor. These fluctuations further induce excess pore pressures and effective stresses that have been recognized as a dominant factor in analyzing the instability of a seabed. When the pore pressure becomes excessive with accompanying decrease in effective stresses, a sedimentary bed may be moved in either horizontal (shear failure) or vertical (liquefaction) directions, then lead to an instability of the seabed (Christian et al., 1974; Jeng, 2001). Therefore, the evaluation of the wave-current induced soil response, including pore pressure, effective stresses and soil displacements, is particularly important for marine geotechnical engineers involved in the design of foundation of offshore installations such as platforms, pipelines and breakwaters.

Two mechanisms of the seabed response have been observed in laboratory and field measurements, depending on the deformation characteristics of seabed (Zen and Yamazaki, 1990). One is caused by the residual or progressive nature of the excess pore pressure, which appears in the initial stage of cyclic loading (Seed and Rahman, 1978). This type of soil response is similar to that induced by earthquakes, caused by the build-up of the excess pore pressure. Another mechanism, caused by the transient or oscillatory excess

\footnotetext{
* Corresponding author at: Center for Marine Geotechnical Engineering, Department of Civil Engineering, Key Laboratory of Ocean Engineering, Shanghai Jiao Tong University, Shanghai 200240, China. Tel.: + 441382386141 ; fax: +44 1382384816.

E-mail addresses: 532183968@qq.com (Y. Zhang), d.jeng@dundee.ac.uk

(D.-S. Jeng), fpgao@imech.ac.cn (F.P. Gao), jszhang@hhu.edu.cn (J.-S. Zhang).
}

pore pressures, and accompanied by the damping of amplitude and phase lag in the pore pressure, appears periodically to each wave (Yamamoto et al., 1978). In this study, only the oscillatory mechanism will be considered. More clarification of two mechanisms can be found in Jeng and Seymour (2007).

Numerous investigations of the oscillatory seabed response of seabed under wave loading have been carried out, based on Biot's poro-elastic theory since the 1970s. Among these, Yamamoto et al. (1978) derived an analytical solution for an isotropic, poroelastic and infinite seabed by treating the pore water and seabed as compressible and deformable medium. Later, Hsu and Jeng (1994) further extended the framework to the unsaturated, isotropic seabed with finite thickness under three-dimensional short-crested waves loading. Numerical modeling is another efficient tool to investigate the transient response of seabed under wave loading. Thomas $(1989,1995)$ proposed a onedimensional finite element model to investigate the waveinduced soil response in a layered seabed. Later, Jeng and Lin (1996) further extended the numerical model for the waveinduced soil response in a porous seabed with variable permeability and shear modulus along burial depth. The seabed soil is unsaturated and hydraulically anisotropic, and subjected to a three-dimensional wave system. However, all these numerical models only considered the wave loading. A detailed review of previous relevant research can be found in Jeng (2003).

In the real ocean environments, ocean waves and currents generally exist simultaneously. However, most previous investigations have considered wave loading only. However, ocean currents affect the seabed response is far from understanding. In fact, according to the 
potential flow theory, the pressures acting on a seabed will be affected significantly by currents. Therefore, it is necessary to understand the influence of current on the seabed response in marine sediments.

The aim of this study is to examine the effects of currents on the seabed response. An analytical solution for the wave-current induced soil response in an infinite porous seabed is proposed. The boundary value problem, including the third-order approximation of nonlinear wave-current interactions, governing equations and boundary conditions for a porous seabed, is outlined in Section 2. Then, an analytical solution for the soil response will be presented in Section 3. Based on the analytical solution, a parametric study will be carried out to examine the effects of currents on the soil response and liquefaction potential in Section 4 and followed by conclusions.

\section{Boundary value problem}

\subsection{Wave-current interactions}

The co-existence of waves and currents in offshore environments is a common phenomenon and their interactions is one of important topics in the practices of coastal engineering. The presence of a current in a propagating wave will change the original characteristics of waves. For example, the following current (currents with the same direction of waves) will elongate the wave length; and the opposing current (currents with the opposite direction of waves) will shorten the wave length. In this study, to obtain more accurate results of seabed response under combined wave and current loadings, the third-order solution of wave-current interactions (Hsu et al., 2009) is used to determine the dynamic wave pressures acting on the seabed.

In this study, we consider a train of monochromatic waves of frequency $(\omega)$ traveling over seafloor, together with a uniform current of velocity $\left(u_{0}\right)$, as depicted in Fig. 1 . The sea water is considered as an incompressible and in-viscid fluid and the flow is irrotational. The flow field of sea water can be described by Laplace's equation,

$\nabla^{2} \phi=\frac{\partial^{2} \phi}{\partial x^{2}}+\frac{\partial^{2} \phi}{\partial z^{2}}=0$

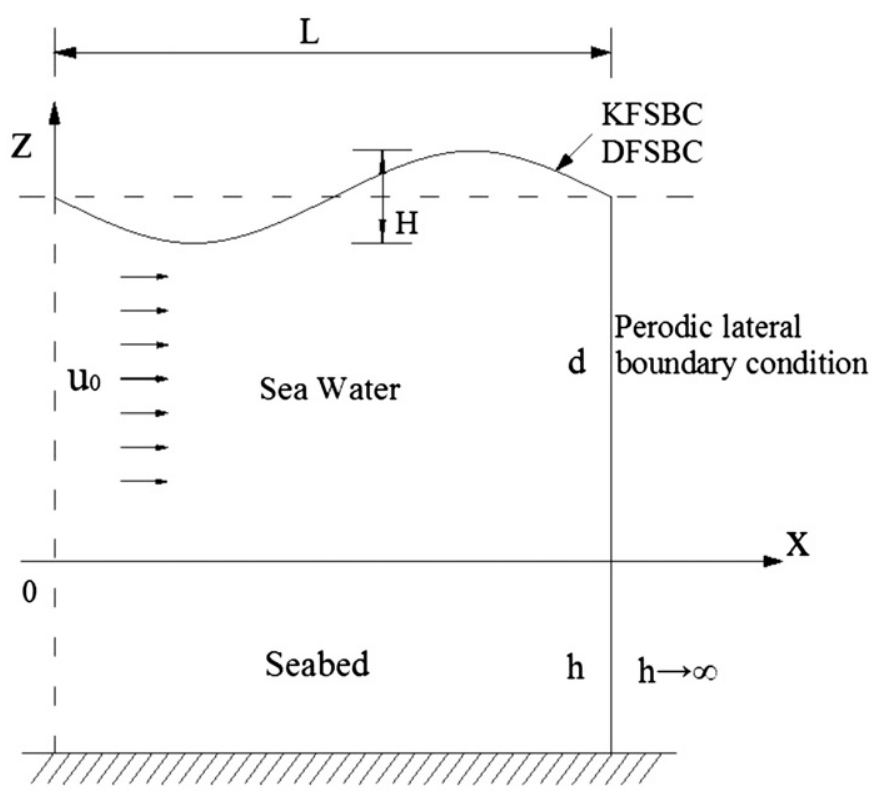

Fig. 1. Definition of wave/current-seabed interaction. where $\phi$ is the velocity potential. The horizontal and vertical velocities of the flow can be formulated as

$u_{f}=-\frac{\partial \phi}{\partial x} \quad$ and $\quad w_{f}=-\frac{\partial \phi}{\partial z}$

where $u_{f}$ and $w_{f}$ are the horizontal and vertical velocities of sea water in flow field, respectively.

The dynamic and kinematic boundary conditions at the free surface $(z=d+\eta)$ can be expressed as,

$-\frac{\partial \phi}{\partial t}+\frac{1}{2}\left(\phi_{x}^{2}+\phi_{z}^{2}\right)+g \eta=B \quad$ at $z=d+\eta$

$\frac{\partial \eta}{\partial t}-\frac{\partial \phi}{\partial x} \frac{\partial \eta}{\partial x}+\frac{\partial \phi}{\partial z}=0 \quad$ at $z=d+\eta$

where $d$ is water depth, $\eta$ is the elevation of free surface relative to the static water level; $B$ is the Bernoulli's constant. The bottom of fluid domain is considered as impermeable

$\frac{\partial \phi}{\partial z}=0 \quad$ at $z=0$

Using the perturbation technique, the third-order approximation for the wave-current interactions (Hsu et al., 2009) is summarized here

$$
\begin{aligned}
\phi(x, z, t)= & -u_{0} x+\frac{H g \cosh k z}{2 \lambda_{*} \cosh k d} \sin \theta+\frac{3 H^{2} \lambda_{*} \cosh 2 k z}{32 \mathrm{sh}^{4}} \sin 2 \theta \\
& +\frac{3 k H^{3}}{512} \lambda_{*} \xi_{1} \cosh 3 k z \sin 3 \theta \\
\eta(x, t)= & \frac{H}{2} \cos \theta+\frac{k H^{2}\left(3+2 \operatorname{sh}^{2}\right) \cosh k d}{16} \frac{\operatorname{sh}^{3}}{\cos 2 \theta} \\
& +\frac{k^{2} H^{3}}{512} \frac{\left(3+14 \mathrm{sh}^{2}+2 \mathrm{sh}^{4}\right)}{\mathrm{sh}^{4}} \cos (k x-\omega t)+\frac{k^{2} H^{3}}{512} \xi_{2} \cos 3 \theta
\end{aligned}
$$

$B(t)=\frac{u_{0}^{2}}{2}-\frac{H^{2}}{16} \frac{\lambda_{*}^{2}}{\mathrm{sh}^{2}}$

$\xi_{1}=\frac{\left(9-4 \mathrm{sh}^{2}\right)}{\mathrm{sh}^{7}}$ and $\xi_{2}=\frac{3\left(9+24 \mathrm{sh}^{2}+24 \mathrm{sh}^{4}+8 \mathrm{sh}^{6}\right)}{\mathrm{sh}^{6}}$

where $\theta=k x-\omega t ; \operatorname{sh}=\sinh k d ; \lambda_{*}=\left(u_{0} k-\omega_{0}\right), H$ is the wave height, $k$ is the wave number, $d$ is the water depth, $u_{0}$ is the current velocity, $g$ is the gravitational acceleration, and the dispersion relation is given by,

$\omega=\omega_{0}+(k H)^{2} \omega_{2}$

where $\omega_{0}=u_{0} k+\sqrt{g k \tanh k d}$ and

$\omega_{2}=-\frac{\left(9+8 \operatorname{sh}^{2}+8 \operatorname{sh}^{4}\right)}{64 \mathrm{sh}^{4}} \lambda *$

The dynamic wave pressure acting on the seabed can be expressed as

$$
\begin{aligned}
P_{b}(x, t)= & \frac{\rho_{f} g H}{2 \cosh k d}\left(1-\frac{\omega_{2} k^{2} H^{2}}{2 \lambda_{*}}\right) \cos \theta-\frac{3 \rho_{f} H^{2}}{8}\left(\frac{\omega_{0} \lambda_{*}}{2 \operatorname{sh}^{4}}+\frac{g k}{3 \sinh 2 k d}\right) \\
& \times \cos 2 \theta-\frac{3 \rho_{f} k H^{3} \omega_{0} \lambda_{*}\left(9-4 \mathrm{sh}^{2}\right)}{512 \operatorname{sh}^{7}} \cos 3 \theta \\
= & P_{1} \cos \theta+P_{2} \cos 2 \theta+P_{3} \cos 3 \theta
\end{aligned}
$$

where $\rho_{f}$ is the density of sea water. When there is no current (i.e., $u_{0}=0 \mathrm{~m} / \mathrm{s}$ ), the above third-order solution can be reduced to the classic form of the solution of third-order nonlinear waves. 


\subsection{Governing equations}

In this study, as shown in Fig. 1, the seabed is treated as an elastic, isotropic and homogeneous porous medium. The consolidation equation (Biot, 1941; Verruijt, 1969), which has been generally accepted as the governing equation for flow of compressible pore fluid in a compressive porous medium, is adopted to treat wave/ current-seabed interactions in a porous seabed as,

$K\left(\frac{\partial^{2} p}{\partial x^{2}}+\frac{\partial^{2} p}{\partial z^{2}}\right)-\gamma_{w} n \beta \frac{\partial p}{\partial t}=\gamma_{w} \frac{\partial z . e p s i}{\partial t}$

where $n$ is soil porosity; $p$ is the pore water pressure; $K$ denotes the soil permeability; and z.epsi; is the volumetric strain.

Neglecting the body forces and the inertia term, the equations governing the overall equilibrium of a porous medium can be expressed as

$\frac{\partial \sigma_{x}^{\prime}}{\partial x}+\frac{\partial \tau_{x z}}{\partial z}=\frac{\partial p}{\partial x}, \quad \frac{\partial \tau_{x z}}{\partial x}+\frac{\partial \sigma_{z}^{\prime}}{\partial z}=\frac{\partial p}{\partial z}$

where $\sigma_{x}^{\prime}$ and $\sigma_{z}^{\prime}$ are effective normal stresses in the horizontal and vertical directions, respectively; $\tau_{x z}$ is shear stress. Herein, compressive normal stresses are denoted as negative.

In Eq. (13), the compressibility of pore fluid $(\beta)$ and the volume strain (z.epsi;) are defined as

$\beta=\left(\frac{1}{K_{f}}+\frac{1-S_{r}}{p_{w 0}}\right), \quad$ z.epsi $;=\frac{\partial u}{\partial x}+\frac{\partial w}{\partial z}$

where $(u, w)$ are the soil displacements in the horizontal and vertical directions, respectively; $S_{r}$ denotes the degree of saturation of seabed, $p_{w 0}$ is the absolute static pressure and $K_{f}$ is the bulk modulus of pore water $\left(K_{f}=2 \times 10^{9} \mathrm{~N} / \mathrm{m}^{2}\right.$; Yamamoto et al., $1978)$. For a fully saturated seabed, $\beta=1 / K_{f}$.

In this study, the linear poro-elastic constitutive model is adopted for the transient seabed response. Under the condition of plane strain, the stress-strain relationships are given as

$\sigma_{x}^{\prime}=2 G\left[\frac{\partial u}{\partial x}+\frac{\mu z . e p s i ;}{1-2 \mu}\right], \quad \sigma_{z}^{\prime}=2 G\left[\frac{\partial w}{\partial z}+\frac{\mu z . e p s i ;}{1-2 \mu}\right]$,

$\tau_{x z}=G\left[\frac{\partial u}{\partial z}+\frac{\partial w}{\partial x}\right]$

where $G$ is the shear modulus of soil; and $\mu$ is the Poisson's ratio.

Substituting (16) into (14), the governing equation for the equilibrium of a porous medium can be expressed as

$G \nabla^{2} u+\frac{G}{(1-2 \mu)} \frac{\partial z \cdot e p s i}{\partial x}=\frac{\partial p}{\partial x} \quad$ and $G \nabla^{2} w+\frac{G}{(1-2 \mu)} \frac{\partial z \cdot e p s i}{\partial z}=\frac{\partial p}{\partial z}$

\subsection{Boundary conditions}

To solve the governing Eqs. (13) and (17), several boundary conditions will be applied. First, the bottom of a seabed is considered to be rigid and impermeable. Therefore, there is no displacement and vertical flow at this bottom. For an infinite seabed,

$u=w=p=0 \quad$ as $z \rightarrow-\infty$

Second, the boundary conditions along the surface of the seabed $(z=0)$ can be expressed as,

$p(x, z=0, t)=P_{b}(x, t), \quad \sigma_{z}^{\prime}=\tau_{x z}=0$

It is noted that the present model is based on the quasi-static Biot's consolidation equations (Biot, 1941), in which the acceleration of soil displacement and relative displacement of pore fluid to soil displacement are ignored. Recently, some analytical solutions of dynamic response for a poro-elastic, isotropic seabed under wave loading based on " $u-p$ " approximation and "full- dynamic approximation" were proposed (Jeng et al., 1999; Jeng and Cha, 2003). A further extended version of analytical solutions was proposed by Ulker et al. (2009), in which the applicable range of all models were identified for the case of wave loading only.

\section{Analytical solution}

Following the framework proposed by Madsen (1978), the soil displacements and pore pressures can be expressed as

$\left\{\begin{array}{c}p \\ u \\ w\end{array}\right\}=\sum_{m=1}^{3} P_{m} \operatorname{Re}\left\{\begin{array}{c}P_{1}^{(m)}(z) \\ U_{1}^{(m)}(z) \\ W_{1}^{(m)}(z)\end{array}\right\} e^{i m(k x-\omega t)}$

where Re denotes the real part of the function and $i=\sqrt{-1}$ denotes the complex variable.

Substituting (20) into (13) and (17), renders the final form of the governing equation as

$\left(D^{2}-m^{2} k^{2}\right)\left(D^{2}-\delta_{m}^{2}\right) U_{1}^{(m)}=0$

$\delta_{m}^{2}=m^{2} k^{2} \frac{K_{x}}{K_{z}}-\frac{i m \omega \gamma_{w}}{K_{z}}\left(n \beta+\frac{1-2 \mu}{2 G(1-\mu)}\right)$

where $K_{x}$ and $K_{z}$ denote the soil permeability in the $x$ and $z$ directions respectively.

The general solution of (21) can be expressed as

$U_{1}^{(m)}=\left(A_{1}+A_{2} z\right) e^{m k z}+\left(A_{3}+A_{4} z\right) e^{-m k z}+A_{5} e^{\delta_{m} z}+A_{6} e^{-\delta_{m} z}$

Considering the bottom boundary condition (18), $A_{3}=A_{4}=$ $A_{6}=0$, which leads to

$U_{1}^{(m)}=\left(A_{1}+A_{2} z\right) e^{m k z}+A_{5} e^{\delta_{m} z}$

Three unknown coefficients, $A_{1}, A_{2}$ and $A_{5}$ can be determined by the boundary condition at the seabed surface (19). Then, the soil displacements and pore pressures can be expressed as

$u=\sum_{m=1}^{3} \frac{i P_{m}}{2 G}\left[\left(C_{m 0}+C_{m 1} z\right) e^{m k z}+C_{m 2} e^{\delta_{m} z}\right] e^{i m(k x-\omega t)}$

$w=\sum_{m=0}^{3} \frac{P_{m}}{2 G}\left[\left(C_{0 m}-\frac{1+2 \lambda_{m}}{m k} C_{1 m}+C_{1 m} z\right) e^{m k z}+\frac{\delta_{m}}{m k} C_{2 m} e^{\delta_{m} z}\right] e^{i m(k x-\omega t)}$

$p=\sum_{m=0}^{3} \frac{P_{m}}{1-2 \mu}\left[\left(1-2 \mu-\lambda_{m}\right) C_{1 m} e^{m k z}+\frac{\delta_{m}^{2}-m^{2} k^{2}}{m k}(1-\mu) C_{2 m} e^{\delta_{m} z}\right] e^{i m(k x-\omega t)}$

where $P_{m}(m=1,2,3)$ are given in (12), and the parameter $\lambda_{m}$ are coupled with soil properties and wave characteristics, as given by Eq. (28).

$\lambda_{m}=\frac{(1-2 \mu)\left[m^{2} k^{2}\left(1-K_{x} / K_{z}\right)+i m \omega \gamma_{w} n \beta / K_{z}\right]}{m^{2} k^{2}\left(1-K_{x} / K_{z}\right)+i m \omega \gamma_{w}(n \beta+(1-2 \mu) / G) / K_{z}}$

In Eqs. (25)-(27), the $C_{i m}$ coefficients are given by

$C_{0 m}=\frac{-\lambda_{m}\left[\mu\left(\delta_{m}-m k\right)^{2}-\delta_{m}\left(\delta_{m}-2 m k\right)\right]}{m k\left(\delta_{m}-m k\right)\left(\delta_{m}-\delta_{m} \mu+m k \mu+m k \lambda_{m}\right)}$

$C_{1 m}=\frac{\delta_{m}-\delta_{m} \mu+m k \mu}{\delta_{m}-\delta_{m} \mu+m k \mu+m k \lambda_{m}}$

$C_{2 m}=\frac{m k \lambda_{m}}{\left(\delta_{m}-m k\right)\left(\delta_{m}-\delta_{m} \mu+m k \mu+m k \lambda_{m}\right)}$ 
The effective normal stresses can be determined from Eq. (16), rendering

$$
\begin{aligned}
\sigma_{x}^{\prime}= & \sum_{m=1}^{3}-P_{m}\left\{\left(m k\left(C_{0 m}+C_{1 m} z\right)+\frac{2 \mu \lambda_{m}}{1-2 \mu} C_{1 m}\right) e^{m k z}\right. \\
& \left.+\left(m k-\frac{\mu\left(\delta_{m}^{2}-m^{2} k^{2}\right)}{m k(1-2 \mu)}\right) C_{2 m} e^{\delta_{m} z}\right\} e^{i m(k x-\omega t)} \\
\sigma_{z}^{\prime}= & \sum_{m=1}^{3} P_{m}\left\{\left(m k\left(C_{0 m}+C_{1 m} z\right)-\frac{2 \lambda_{m}(1-\mu)}{1-2 \mu} C_{1 m}\right) e^{m k z}\right. \\
& \left.+\frac{\delta_{m}^{2}(1-\mu)-m^{2} k^{2} \mu}{m k(1-2 \mu)} C_{2 m} e^{\delta_{m} z}\right\} e^{i m(k x-\omega t)}
\end{aligned}
$$

and the shear stress is given by

$$
\tau_{x z}=\sum_{m=1}^{3} i P_{m}\left[\left(m k C_{0 m}+\left(m k z-\lambda_{m}\right) C_{1 m}\right) e^{m k z}+\delta_{m} C_{2 m} e^{\delta_{m} z}\right] e^{i m(k x-\omega t)}
$$

\section{Results and discussions}

\subsection{Comparison with experimental data}

In this study, the results of the present solution will be compared with three experimental data. Since most experiments for seabed response under dynamic loadings available in the literature have been limited to wave loading only, the present model will be reduced to the case without currents and compared

a
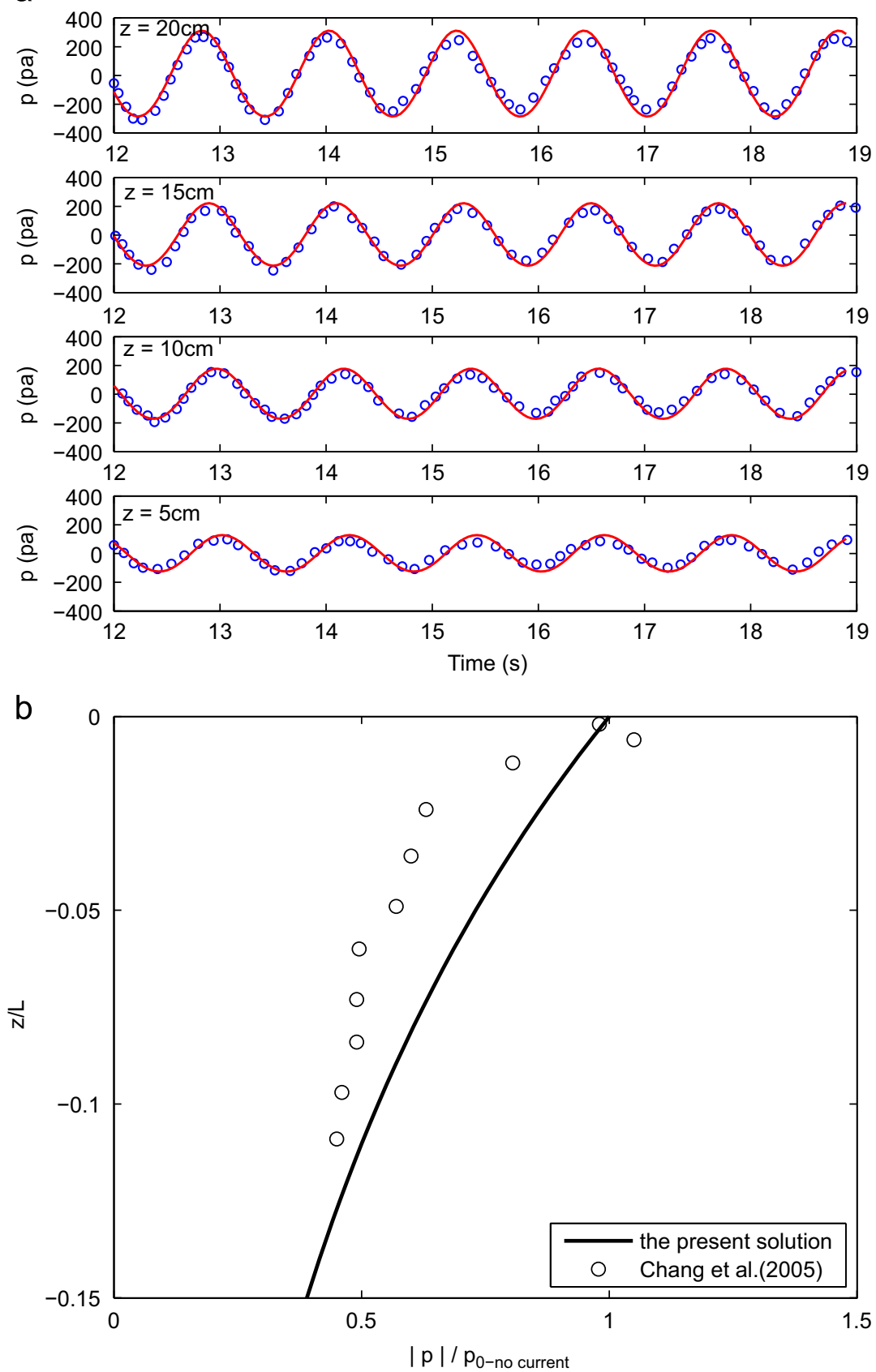

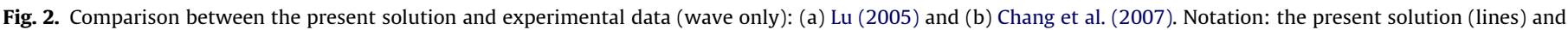
experimental data (symbols). 
with the previous experimental data (Lu, 2005; Chang et al., 2007). Lu (2005) conducted a series of lab experiments about the dynamic response of sand bed to the waves propagating on it in a wave flume which is $60 \mathrm{~m}$ long, $1.5 \mathrm{~m}$ wide and $1.8 \mathrm{high}$. The sand bed is consisted of coarse sand. The pore pressure at the four points on the midline of sand bed are monitored in experiments. The wave characteristics of the regular wave are $H=12 \mathrm{~cm}$, $d=0.4 \mathrm{~m}, T=1.2 \mathrm{~s}$ (Fig. 2a). Herein, the third-order stokes wave theory is adopted to generate the wave-induced dynamic pressures acting on seabed. The properties of coarse sand provided by Lu (2005) are: shear modulus $G=10^{7} \mathrm{~N} / \mathrm{m}^{2}$, Possion's ratio $\mu=0.3$, Permeability $k=10^{-3} \mathrm{~m} / \mathrm{s}$, Porosity $n=0.3893$, the mean size of sand particles $d_{50}=0.44 \mathrm{~mm}$ and Saturation $S_{r}=98 \%$. Another relevant experiment is conducted by Chang et al. (2007). The movable seabed model is illustrated in Fig. 2b. In this experiment, the wave characteristics of the regular wave are $H=25 \mathrm{~cm}, d=0.562 \mathrm{~m}, T=2 \mathrm{~s}$, the Stoke's second-order wave theory is adopted to generate the wave-induced dynamic pressures acting on the seabed. The properties of the coarse sand are taken as Shear modulus $G=10^{7} \mathrm{~N} / \mathrm{m}^{2}$, Possion's ratio $\mu=0.12$, Permeability $k=2.11 \times 10^{-3} \mathrm{~m} / \mathrm{s}$, Porosity $n=0.48$, the mean size of sand particles $d_{50}=0.22 \mathrm{~mm}$ and the degree of saturation $S_{r}=1.0$.

The comparisons of the wave induced dynamic pore pressure at the four points on the mid-line of sand bed between the numerical results and the experimental data of $\mathrm{Lu}$ (2005) are shown in Fig. 2a. As illustrated in the figure, the numerical results predicted by the present analytical solution overall agree with the experimental data (Lu, 2005). The comparisons of Chang et al. (2007)'s experiment and the present numerical results are shown in Fig. 2b. In the figure, the trend of the present solution overall agree with that of Chang et al. (2007). Some differences between the present solution and the experimental data are observed in Fig. $2 \mathrm{~b}$, due to that the present solution is for a infinite seabed but the experiments were in a seabed of finite thickness.

Another comparison with the recent experiment conducted by Qi et al. (2012) for the wave-current induced pore pressure in a porous seabed is presented in Fig. 3. In the experiments, the local scour process and pore pressure within a sandy bed around a mono-pile foundation under combined wave and current loading were measured. To compare with the present solution, we select the measured point far from the mono-pile, which will present the case without a structure. The properties of the seabed provided by Qi et al. (2012) are: shear modulus $G=10^{7} \mathrm{~N} / \mathrm{m}^{2}$, Possion's ratio $\mu=0.3$, Permeability $k=1.88 \times 10^{-4} \mathrm{~m} / \mathrm{s}$, the mean size $\left(d_{50}\right)$ and effective size $\left(d_{10}\right)$ of sand particles are $0.25 \mathrm{~mm}$ and $0.21 \mathrm{~mm}$, respectively; void ratio $(e)$ is 0.771 , and fully saturated. The wave and current characteristics of the data presented in the figure are: water depth $d=0.5 \mathrm{~m}$, wave period $T=1.4 \mathrm{~s}$; wave height $H=0.12 \mathrm{~m}$ and current velocity $u_{0}=0.1 \mathrm{~m} / \mathrm{s}$. As shown in Fig. 3, an overall agreement between the present solution and the experimental data is found.

Table 1

Information of case study.

\begin{tabular}{ll}
\hline Case 1 & Wave loading (linear model) \\
Case 2 & Combined wave and current loading (linear wave model) \\
Case 3 & Wave loading (third-order approximation) \\
Case 4 & Combined wave and current loading (third-order approximation)
\end{tabular}
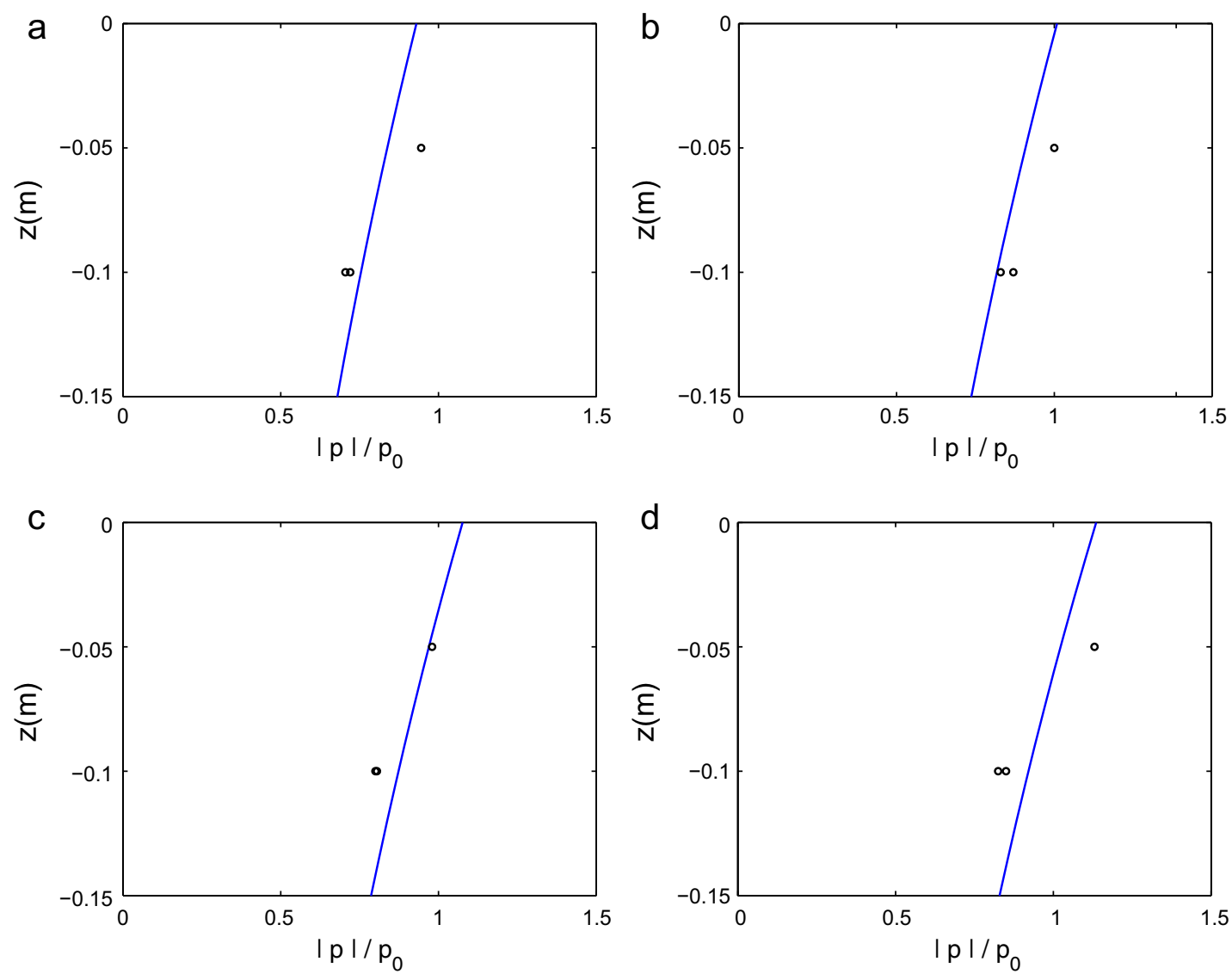

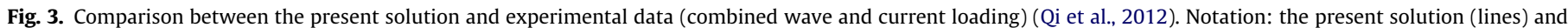
experimental data (symbols). 
Table 2

Input data for numerical examples.

\begin{tabular}{ll}
\hline Wave characteristics & \\
Wave period $(T)$ & $10.0 \mathrm{~s}$ \\
Water depth $(d)$ & $10.0 \mathrm{~m}$ \\
Wave height $(H)$ & $5.0 \mathrm{~m}$ \\
Current velocity $\left(u_{0}\right)$ & $2 \mathrm{~m} / \mathrm{s}$ \\
Seabed characteristics & \\
$\quad$ Permeability for fine sand & $1.0^{-3} \mathrm{~m} / \mathrm{s}$ \\
$\left(K_{x}=K_{z}\right)$ & \\
Permeability for coarse sand & $1.0^{-2} \mathrm{~m} / \mathrm{s}$ \\
$\left(K_{x}=K_{z}\right)$ & \\
Shear modulus $(G)$ & $1.0^{7} \mathrm{~N} / \mathrm{m}^{2}$ \\
Poisson's ratio $(\mu)$ & 0.33 \\
Porosity $\left(n_{e}\right)$ & 0.35 \\
Degree of saturation $\left(S_{r}\right)$ & 0.975
\end{tabular}

a

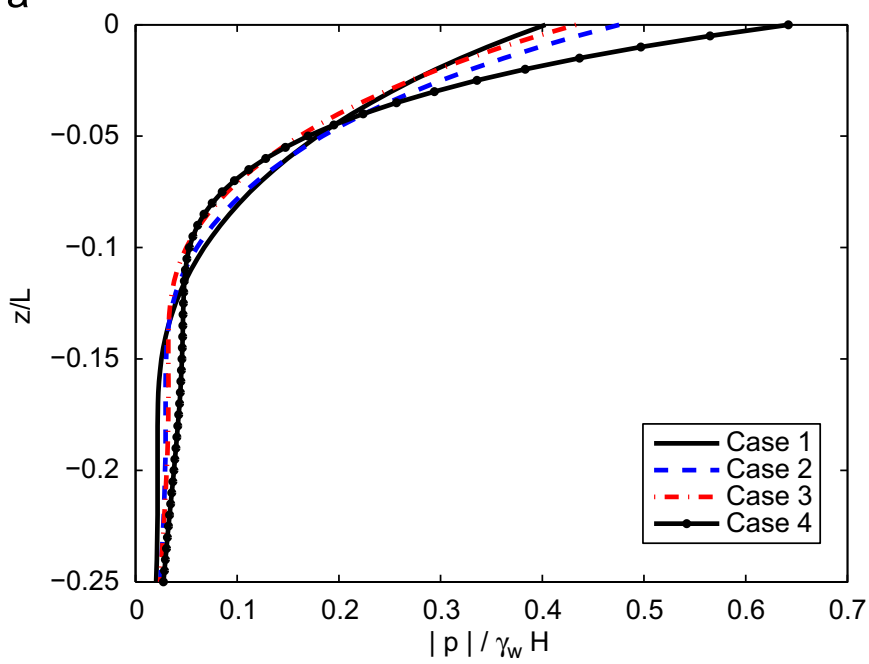

b

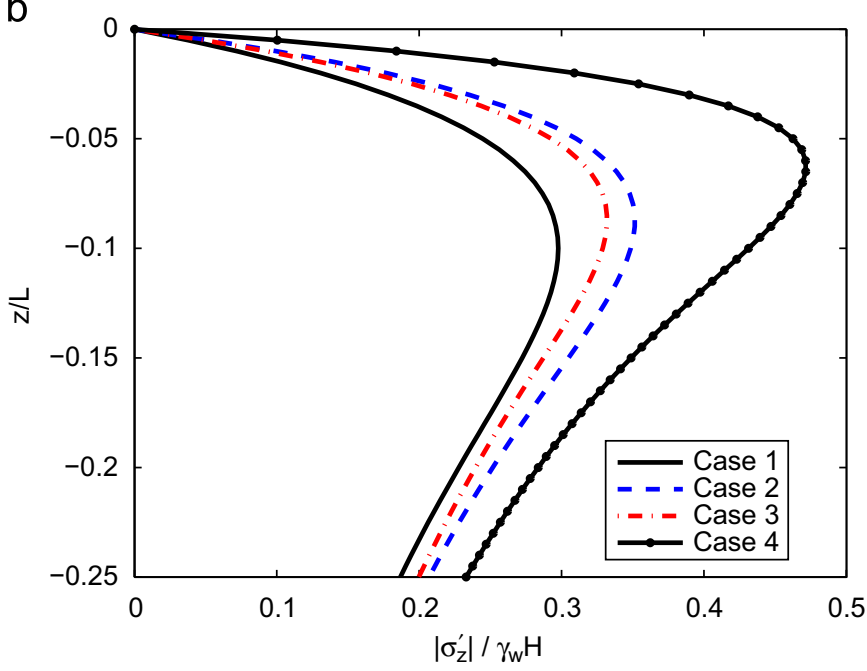

Fig. 4. Vertical distributions of the maximum amplitude of (a) pore pressure $\left(|p| / \gamma_{w} H\right)$ and (b) effective normal stress $\left(\left|\sigma_{z}^{\prime}\right| / \gamma_{w} H\right)$ in coarse sand for four different cases.

\subsection{Seabed response}

The objective of this study is to investigate the effects of wave non-linearity and currents on the seabed response (including pore pressure, effective normal stresses and shear stresses). In this section, four different cases are considered (see Table 1). Among these, Case 1 is the linear wave only, which has been used for most previous study of the wave-induced seabed response (Hsu and Jeng, 1994; Madsen, 1978; Thomas, 1995; Yamamoto et al., 1978; Zen and Yamazaki, 1990). Case 2 is the linear approximation of waves and currents. Case 3 is the third-order approximation of wave loading; while Case 4 is the solution for the nonlinear wave and current interactions. The input data for numerical examples are tabulated in Table 2 .

Fig. 4 illustrates the vertical distributions of the maximum amplitude of seabed response in coarse sand for four different combination of waves and currents. In this example, the currents are in the same direction of waves. As shown in Fig. 4 for coarse sand, the pore pressure for Case 4 (non-linear waves and currents) is much larger than that of Case 1 (linear wave), and the inclusion of currents will enlarge the amplitude of pore pressures. However, this influence is only significant in the region near seabed surface $(|z / L| \leq 0.05)$. Compared with pore pressures, the influences of wave-current nonlinear interactions (Case 4 vs Case 1) on the vertical effective normal stresses and shear stresses are more significant. For example, the maximum difference of $\left|\sigma_{z}^{\prime}\right|$ between Cases 1 and 4 can reach $20 \%$ of $\left(\gamma_{w} H\right)$.

A similar trend for the influences of wave nonlinearity and currents is observed in fine sand (Fig. 5). However, there is a

a

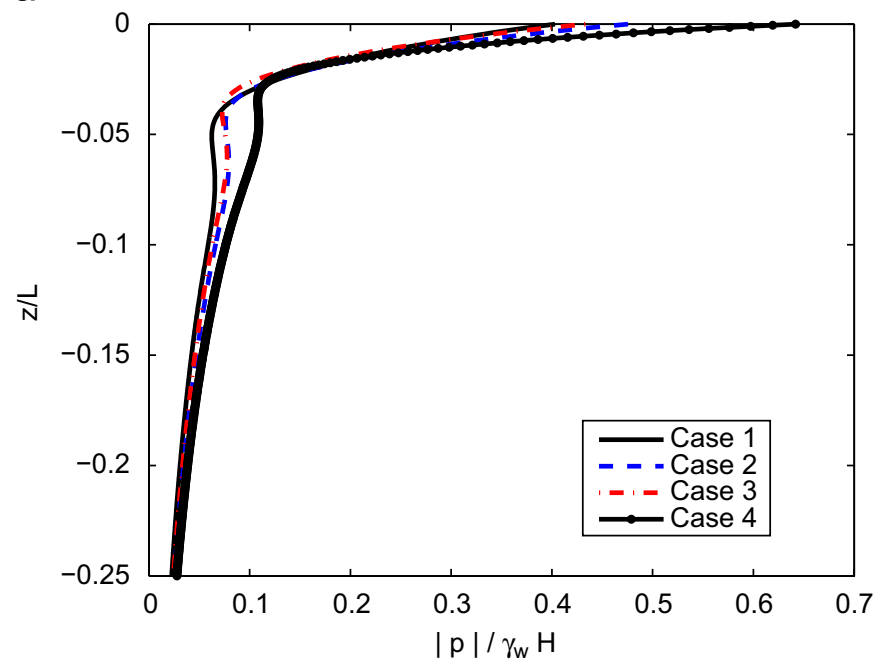

b

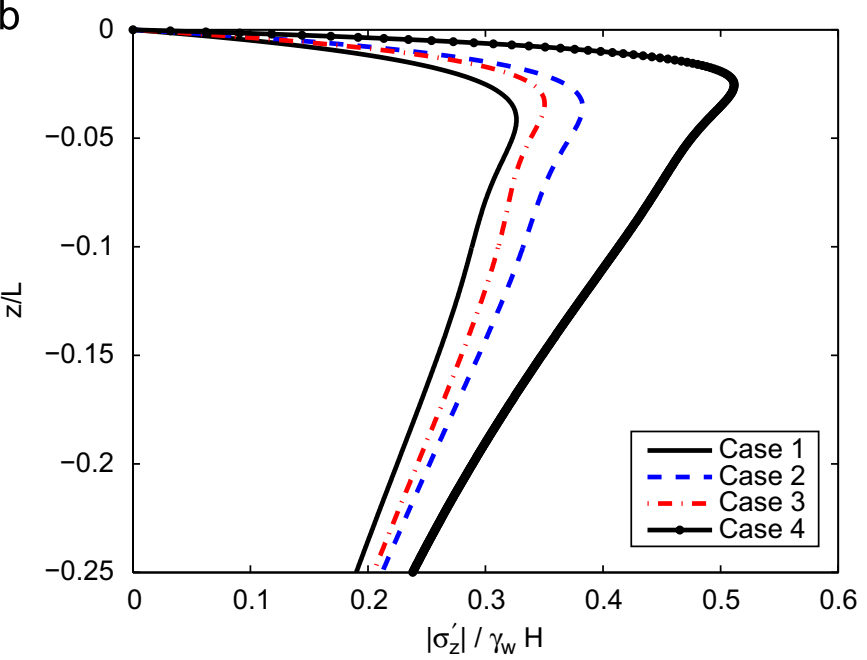

Fig. 5. Vertical distributions of the maximum amplitude of (a) pore pressure $\left(|p| / \gamma_{w} H\right)$ and (b) effective normal stress $\left(\left|\sigma_{z}^{\prime}\right| / \gamma_{w} H\right)$ in fine sand for four different cases. 
a

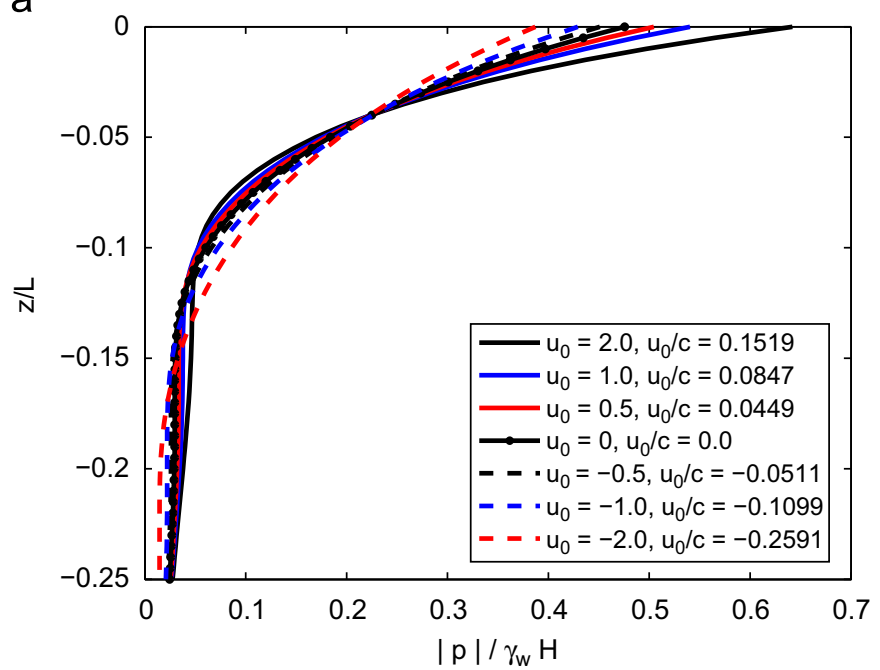

b

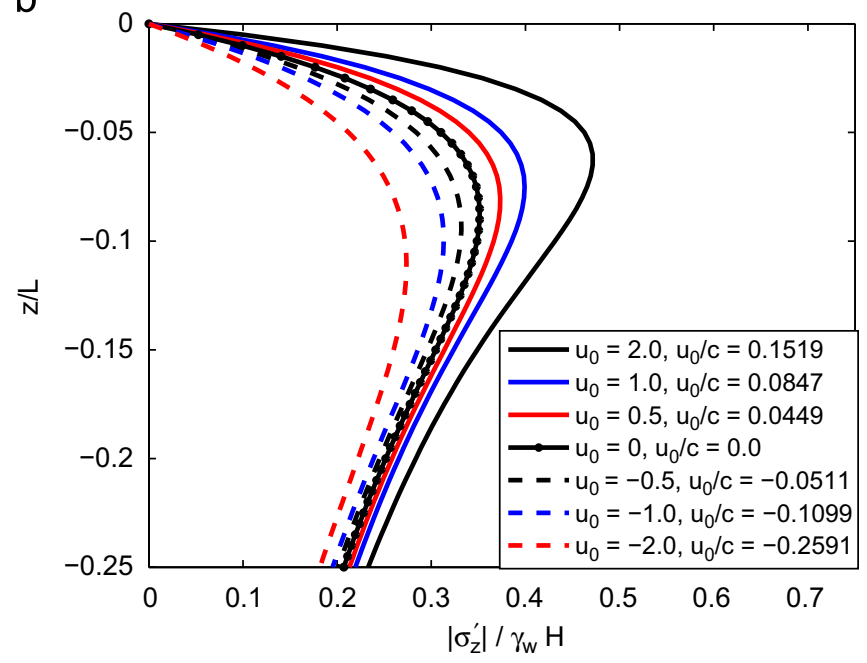

Fig. 6. Vertical distributions of the maximum amplitude of (a) pore pressure $\left(|p| / \gamma_{w} H\right)$ and (b) effective normal stress $\left(\left|\sigma_{z}^{\prime}\right| / \gamma_{w} H\right)$ in coarse sand for various current velocities $\left(u_{0}\right)$.

sudden change of gradient of seabed response near the seabed surface $(z / L=-0.01)$. This unusual results of discontinuity are only observed in fine sand and reported in the previous work (Madsen, 1978). The physical insight of these results needs detailed validations from experiments.

To further examine the effects of currents on the seabed response, Fig. 6 illustrates the results of seabed response for various current velocities in coarse sand. In the numerical examples, a positive current velocity represents the currents and waves being the same direction, while the negative value denotes the currents and waves being an opposite direction. For the case of $u_{0}=0, u_{0} / c=0$, the third-order wave theory is applied (i.e., only non-linear waves are considered). As shown in the figures, the seabed response increases as the current velocity increases for the case of the following current, i.e., waves and currents in the same direction. An opposite trend is observed in the case of opposite currents.

\subsection{Liquefaction}

It has been well-known that the soil will be liquefied when the vertical effective stress vanishes, thus losing its strength to carry any load, and consequently causing seabed instability. Zen and

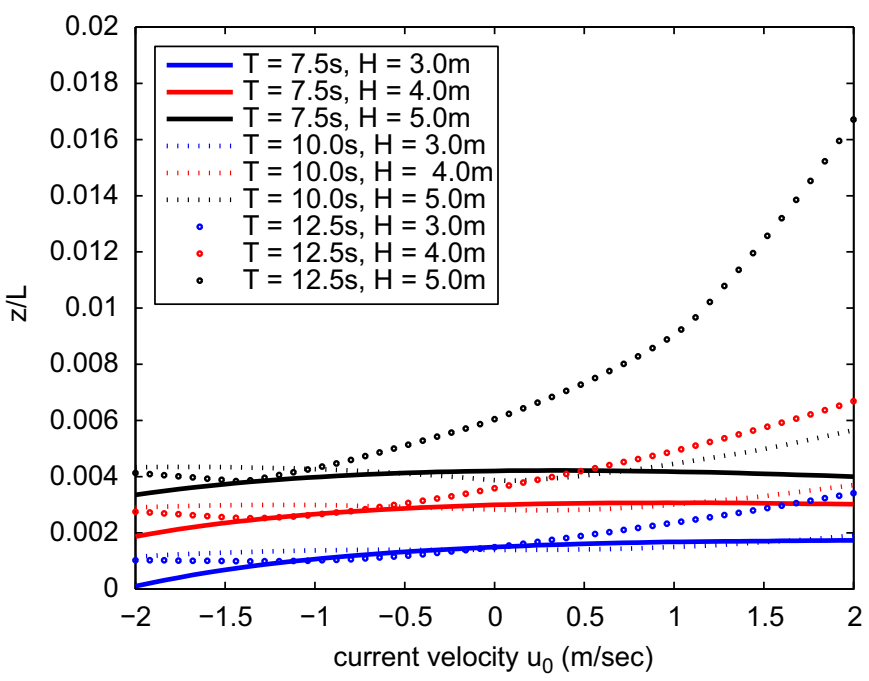

Fig. 7. Distributions of the maximum liquefaction depth versus current velocity $(d=10 \mathrm{~m})$.

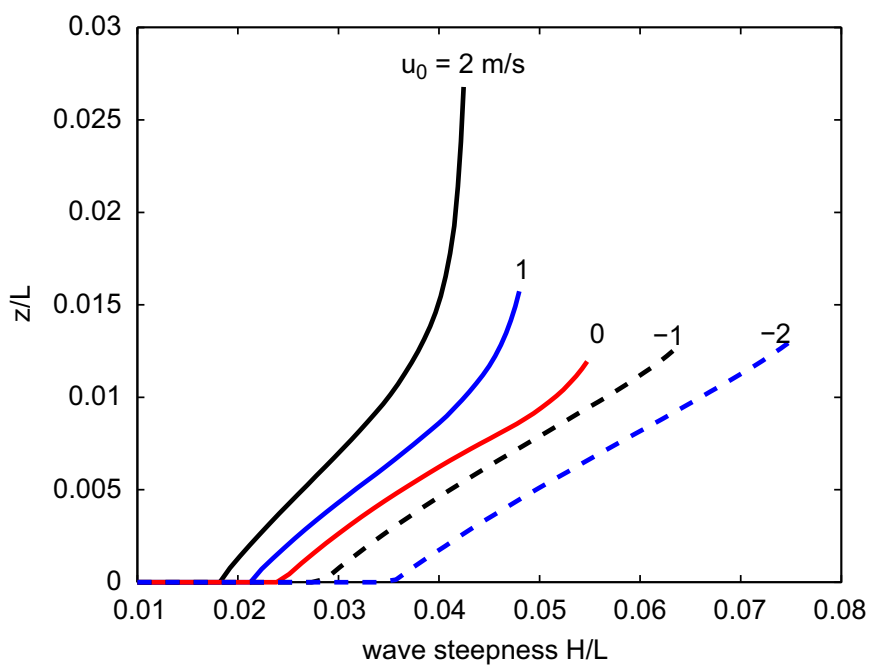

Fig. 8. Distributions of the maximum liquefaction depth versus wave steepness $(H=3 \mathrm{~m}, T=10 \mathrm{~s}, d=10 \mathrm{~m})$.

Yamazaki (1990) suggested that liquefaction occurs, for a 2-D progressive wave, when

$-\left(\gamma_{s}-\gamma_{w}\right) z+\left(P_{b}(x, t)-p(x, z, t)\right) \leq 0$

where $\gamma_{s}$ denotes the bulk specific weight of soil. This equation implies that liquefaction in a seabed may occur when the excess pore pressure becomes greater than the overburden soil pressure.

Based on the above liquefied criterion, Eq. (35), the effects of current velocity on the maximum liquefaction depth $\left(z_{L}\right)$ can be examined. In the numerical example, the current velocity varies between $-2 \mathrm{~m} / \mathrm{s}$ (the opposite direction to the waves) and $2 \mathrm{~m} / \mathrm{s}$ (the following direction to the wave). As shown in Fig. 7, the maximum liquefaction depth increases as the current velocities with a fixed wave height $(H=3 \mathrm{~m})$ and a fixed wave period $(T=10 \mathrm{~s})$. It is noted that the maximum liquefaction depth $\left(z_{L}\right)$ is almost a constant between $u_{0}=-0.5 \mathrm{~m} / \mathrm{s}$ and $u_{0}=0.5 \mathrm{~m} / \mathrm{s}$. Furthermore, a significant change of $z_{L}$ is observed when the current velocity approaches $2 \mathrm{~m} / \mathrm{s}$. Besides, there is a obvious trend that the liquefaction depth increases as the wave height increases. 
To further investigate the influences of currents on the maximum liquefaction depth $\left(z_{L}\right)$, Fig. 8 presents the distribution of $z_{L}$ versus wave steepness for various current velocities. It is noted that the horizontal axis is the wave steepness $(H / L)$, which varies as current velocity and wave height changes. The wavelength will increase for the case of the currents in the same direction of waves, while it will decrease for the case of the currents in the opposite direction of waves. Therefore, the wave steepness will be larger for the case of the opposite currents with the same wave height. It is observed that largest $z / L$ can reach 0.0265 for the case of $u_{0}=2 \mathrm{~m} / \mathrm{s}$, while that for $u_{0}=-2 \mathrm{~m} / \mathrm{s}$ is only 0.013 . It is also noted that the relative maximum liquefaction depth for the case with wave loading only is about 0.012. This implies that the liquefaction depth will be underestimated for the case with following currents, if the conventional model (i.e., without currents) is used.

\section{Conclusions}

In this paper, an analytical solution for seabed response under combined wave and current loadings is proposed. The new contribution of this study is the inclusion of currents into seabed response together with nonlinear wave loadings. In the model, the third-order approximation of the wave-current interactions was employed for the loading along the seabed surface. An overall agreement between the present solution and the existing experimental data was observed. Based on the numerical results, the following conclusions can be drawn.

1. For both coarse and fine sand, the inclusion of the following currents and wave non-linearity (i.e., comparison between Cases 1 and 4) will enlarge the amplitude of pore pressures. This influence is only significant in the region near the seabed surface where liquefaction normally occurs. Furthermore, the effects of currents on vertical effective normal stress is more significant. The maximum difference between Cases 1 and 4 may reach $10 \%$ of static water pressure.

2. Based on the numerical examples presented, the amplitude of seabed response increases as the current velocity increases for the following current (with the same direction as waves). On the other hand, seabed response decrease as the current velocity increase for the opposite currents.

3. Based on the present solution and the liquefaction criterion proposed by Zen and Yamazaki (1990), the maximum liquefaction depth due to combined waves and current loadings were further examined. The maximum liquefaction depth $\left(z_{L}\right)$ increases as current velocity and wave height increase.

In this study, only the oscillatory pore pressure based on Biot's consolidation theory is considered. For more advanced model such as $u-p$ approximation, full dynamic model can be further extended with the basis of the present model.

\section{Acknowledgement}

This study is supported by Shanghai Jiao Tong University State Key Laboratory of Ocean Engineering Self-Development Grant \#GKZD010053 (China), SiChuan University State Key Laboratory of Hydraulics and Mountain River Engineering Open Fund Scheme \#SKLH-OF-1005 (China), EPSRC Grant \#EP/G006482/1 (UK) and National Natural Science Foundation of China Grant \#41176073 and \#51209083 (China).

\section{References}

Biot, M.A., 1941. General theory of three-dimensional consolidation. J. Appl. Phys. 26 (2), 155-164.

Chang, S.C., Lin, J.G., Chien, L.K., Chiu, Y.F., 2007. An experimental study on non-linear progressive wave-induced dynamic stresses in seabed. Ocean Eng. 34, 2311-2329.

Christian, J.T., Taylor, P.K., Yen, J.K.C., Erali, D.R., 1974. Large diameter underwater pipeline for nuclear power plant designed against soil liquefaction. In: Proceeding of Offshore Technology Conference, pp. 597-606.

Hsu, H.C., Chen, Y.Y., Hsu, J.R.C., Tseng, W.J., 2009. Nonlinear water waves on uniform current in Lagrangian coordinates. J. Nonlinear Math. Phys. 16, 47-61.

Hsu, J.R.C., Jeng, D.-S., 1994. Wave-induced soil response in an unsaturated anisotropic seabed of finite thickness. Int. J. Numer. Anal. Methods Geomech. 18 (11), 785-807.

Jeng, D.-S., 2001. Mechanism of the wave-induced seabed response in the vicinity of a breakwater: a review. Ocean Eng. 28 (5), 539-572.

Jeng, D.-S., 2003. Wave-induced sea floor dynamics. Appl. Mech. Rev. 56 (4), 407-429.

Jeng, D.-S., Cha, D.H., 2003. Effects of dynamic soil behavior and wave nonlinearity on the wave-induced pore pressure and effective stresses in porous seabed. Ocean Eng. 30 (16), 2065-2089.

Jeng, D.-S., Lin, Y.S., 1996. Finite element modelling for water waves-soil interaction. Soil Dyn. Earthquake Eng. 15 (5), 283-300.

Jeng, D.-S., Rahman, M.S., Lee, T.L., 1999. Effects of inertia forces on wave-induced seabed response. Int. J. Offshore Polar Eng. 9 (4), 307-313.

Jeng, D.-S., Seymour, B.R., 2007. A simplified analytical approximation for porewater pressure build-up in a porous seabed. J. Waterway Port Coastal Ocean Eng. ASCE 133 (4), 309-312.

Lu, H.B., 2005. The Research on Pore Water Pressure Response to Waves in Sandy Seabed. Ph.D. Thesis, Changsha University of Science \& Technology, Changsha, Hunan, China.

Madsen, O.S., 1978. Wave-induced pore pressures and effective stresses in a porous bed. Géotechnique 28 (4), 377-393.

Qi, W., Gao, F., Han, X., Gong, Q., 2012. Local scour and pore-water pressure around a monopile foundation under combined waves and currents. In: Proceedings of the Twenty-Second (2012) International Offshore and Polar Engineering Conference (ISOPE2012), pp. 159-165.

Seed, H.B., Rahman, M.S., 1978. Wave-induced pore pressure in relation to ocean floor stability of cohesionless soils. Mar. Geotechnol. 3 (2), 123-150.

Thomas, S.D., 1989. A finite element model for the analysis of wave induced stresses, displacements and pore pressure in an unsaturated seabed. I: theory. Comput. Geotechnics 8 (1), 1-38.

Thomas, S.D., 1995. A finite element model for the analysis of wave induced stresses, displacements and pore pressure in an unsaturated seabed. II: model verification. Comput. Geotechnics 17 (1), 107-132.

Ulker, M.B.C., Rahman, M.S., Jeng, D.-S., 2009. Wave-induced response of seabed: various formulations and their applicability. Appl. Ocean Res. 31 (1), 12-24.

Verruijt, A., 1969. In: De Wiest, R.J.M. (Ed.), Elastic Storage of Aquifers. Academic Press, New York, pp. 331-376, Chapter 8.

Yamamoto, T., Koning, H., Sellmeijer, H., Hijum, E.V., 1978. On the response of a poro-elastic bed to water waves. J. Fluid Mech. 87 (1), 193-206.

Zen, K., Yamazaki, H., 1990. Mechanism of wave-induced liquefaction and densification in seabed. Soils Found. 30 (4), 90-104. 